\title{
On the Trade-off Between Feedback and Capacity in Measured MU-MIMO Channels
}

\author{
Florian Kaltenberger, Marios Kountouris, David Gesbert, Raymond Knopp
}

\begin{abstract}
In this work we study the capacity of multi-user multiple-input multiple-output (MU-MIMO) downlink channels with codebook-based limited feedback using real measurement data. Several aspects of MU-MIMO channels are evaluated. Firstly, we compare the sum rate of different MU-MIMO precoding schemes in various channel conditions. Secondly, we study the effect of different codebooks on the performance of limited feedback MU-MIMO. Thirdly, we relate the required feedback rate with the achievable rate on the downlink channel. Real multi-user channel measurement data acquired with the Eurecom MIMO OpenAir Sounder (EMOS) is used.

To the best of our knowledge, these are the first measurement results giving evidence of how MU-MIMO precoding schemes depend on the precoding scheme, channel characteristics, user separation, and codebook. For example, we show that having a large user separation as well as codebooks adapted to the second order statistics of the channel gives a sum rate close to the theoretical limit. A small user separation due to bad scheduling or a poorly adapted codebook on the other hand can impair the gain brought by MU-MIMO. The tools and the analysis presented in this paper allow the system designer to trade-off downlink rate with feedback rate by carefully choosing the codebook.
\end{abstract}

Index Terms-Multi-user MIMO systems, Limited Feedback, Information rates, Radio propagation, Channel sounding

\section{INTRODUCTION}

Multiple-input multiple-output (MIMO) wireless communication systems can substantially improve the spectral efficiency in wireless point-to-point links. Early theoretical results $[1,2]$ are beginning to be successfully implemented in systems and standards $[3,4]$.

Recently there has also been a great deal of interest on how to carry these performance gains over to the system level. Multi-user MIMO (MU-MIMO) refers to a system where a transmitter equipped with multiple antennas is communicating with several users simultaneously on the same physical resources. The users can have multiple antennas too, but this is not a necessity. Especially the downlink (or broadcast) channel of such systems has received a lot of attention in the context of

Original version submitted 19-Dec-2008, revised 16-Apr-2009, accepted 25-May-2009.

Florian Kaltenberger, David Gesbert, and Raymond Knopp are with Eurecom, 2229, Route des Cretes - B.P. 193, 06904 Sophia Antipolis, France (email: florian.kaltenberger@eurecom.fr), Marios Kountouris is with the Wireless Networking and Communications Group, the University of Texas at Austin, Texas, USA.

Parts of this work have been presented at the IEEE Global Communications Conference (GLOBECOM 2008), New Orleans, USA, Nov.-Dec. 2008. This research was supported by the European Commission through the projects CHORIST, SENDORA, and NEWCOM++ as well as by Eurecom through its industrial partners Swisscom, Thales, SFR, Orange France, STMicroelectronics, Sharp, Cisco, and BMW Group. emerging cellular systems, such as the IEEE worldwide interoperability for microwave access (WiMAX) [5] or the 3GPP long term evolution (LTE) [4]. The downlink channel is also the focus of this paper.

By regarding the set of antennas of the users as one virtual antenna array, results from conventional MIMO can be readily applied to the MU-MIMO case. However, since the users cannot cooperate, all the space-time processing has to be done at the transmitter side in the form of precoding. The performance of MU-MIMO depends on a variety of factors such as (i) the precoding scheme used, (ii) the quality of the channel state information at the transmitter, and (iii) the channel characteristics and the user separation.

Information theory reveals that if there is full channel state information at the transmitter (CSIT) and the receiver (CSIR), the optimum transmit strategy for the MU-MIMO broadcast channel involves a theoretical pre-interference cancellation technique known as dirty paper coding (DPC) combined with an implicit user scheduling and power loading algorithm [6, 7]. Since DPC is computationally expensive and hard to implement also simpler, sub-optimal transmit strategies based on user scheduling together with linear precoding have been proposed $[8,9]$.

CSIT can be achieved either by exploiting channel reciprocity in a time division duplex (TDD) system or by means of a limited feedback channel in a frequency division duplex (FDD) system. In the latter case, which is also the focus of this paper, channel vector quantization (CVQ) based on predefined codebooks can be used to feed back a quantized version of the channel [10]. The codebook has to be designed in a way to minimize the quantization error of the channel matrices as well as the feedback overhead. However, minimizing the quantization error requires large codebooks which require a large amount of feedback [11]. In a real system, the best tradeoff between these two design criteria has to be found.

Most of the current literature studies MU-MIMO systems in ideal simulation environments using independent and identically distributed (i.i.d.) Rayleigh fading channel models. Compared to a single-user MIMO (SU-MIMO) time division multiple access (TDMA) system, DPC with perfect CSIT can bring a theoretical performance gain of up to $\max (\min (M / N, K), 1)$ in an i.i.d. Rayleigh fading channel, where $M$ and $N$ is the number of transmit antennas and receive antennas respectively and $K$ is the number of users [12]. If all users experience the same transmit correlation matrix and the number of users is large, [13] showed that the rate loss due to correlation is $M \log c$, where $c$ depends on the scheduling scheme and the eigenvalues of the covariance 
matrix

However it was shown in that neither the i.i.d. assumption nor the assumption of a common transmit correlation matrix for one user holds true in real measured MU-MIMO channels $[14,15]$. From a system level perspective it is interresting to see the performance of MU-MIMO with limited feedback in realistic conditions with a small number of users.

Thus, in this paper we use real channel measurements to study MU-MIMO systems with $K=M$ users. We compare the performance of different linear MU-MIMO precoding schemes, such as zero-forcing (ZF) and regularized channel inversion (also called Minimum Mean Square Error (MMSE) precoder) [8] with the achievable capacity in such channels (the DPC region) [16]. We also study the impact of limited feedback based on CVQ using a Fourier codebook, a Grassmannian codebook, a random codebook, and a random codebook exploiting the second order statistics of the channel. Last but not least we evaluate the information rate on the feedback channel using a first order Markov chain model for the temporal evolution of the feedback [17]. Relating this feedback rate to the achievable rate on the broadcast channel provides a mean to evaluate different codebooks.

MU-MIMO channel measurements have been obtained using the Eurecom MIMO OpenAir Sounder (EMOS) [18]. The EMOS can perform real-time channel measurements synchronously over multiple users moving at vehicular speed. The measured channels are stored to disk for offline analysis. For this paper, we have used four transmit antennas and four users with one antenna each.

Related Work: Many measurement campaigns for pointto-point MIMO channels have been carried out to date, but MU-MIMO measurements are still rare in the literature. A common practice is to conduct SU-MIMO measurements and later combine them into a MU-MIMO channel. Such an approach has for example been reported in [19-22]. Recently, [23] has claimed that under certain conditions this method is feasible. However, this is definitely not the case for rapidly changing environments and the high-mobility measurements as considered in this paper. To the best of the authors knowledge, real synchronized MU-MIMO channel measurements have only been described in [24]. The measurements were conducted using a MEDAV-LUND channel sounder with its corresponding receiver as well as the receiver of an Elektrobit channel sounder. The measurements of the two receivers are synchronized in a post-processing step using a dummy channel that was inserted between snapshots. The authors present capacity results for the uplink channel, as well as path-loss and delay spreads for the measured scenarios. However, the measurements in [24] are limited to a two-user case while also being very costly and time-consuming.

Contributions and Outline: The contributions of the paper are as follows

- We assess the performance of different MU-MIMO schemes in using several different channel measurements.

- We study the effect of limited feedback using different codebooks in real world conditions.

- We introduce a novel way how to relate feedback rate to capacity of MU-MIMO channels thus providing a mean

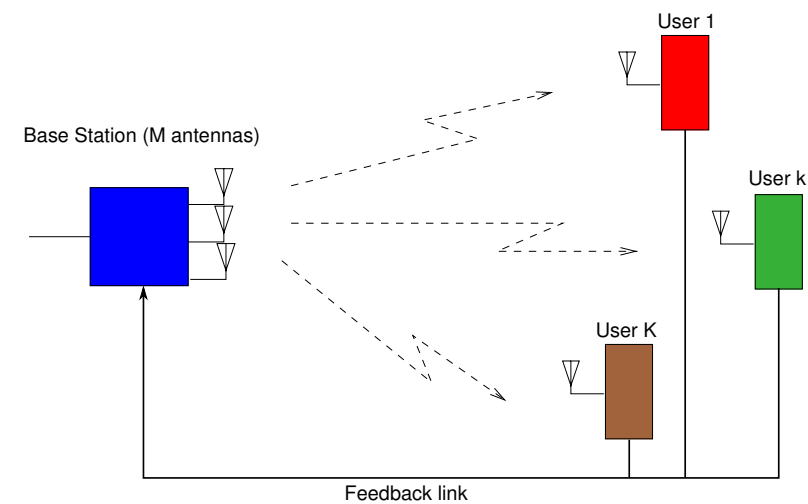

Fig. 1. Multi-user MIMO System Model.

to evaluate different codebooks.

- We present the EMOS platform and show how the challenge of performing MU-MIMO measurements synchronously over multiple users can be addressed.

The paper is organized as follows. In Section II we describe the MU-MIMO system model. Section III reviews results on the capacity of MU-MIMO channels and describes the different linear precoding schemes studied in this paper. In Section IV we describe how to obtain partial CSIT by means of limited feedback. The measurements and their results are discussed in Section V. Finally conclusions are drawn in Section VI. The Eurecom MIMO OpenAir Sounder (EMOS) is described in the Appendix.

Notation: Column vectors and matrices are denoted by a and $\mathbf{A}$ respectively. $\mathbf{I}_{M}$ is the identity matrix of size $M$ and $\mathbf{0}_{M}$ is an $M$-dimensional vector of zeros. The Euclidean $\left(\ell_{2}\right)$ norm of a vector $\mathbf{a}$ is denoted by $\|\mathbf{a}\|$ and the Frobenius norm of a matrix $\mathbf{A}$ is denoted by $\|\mathbf{A}\|_{F}$. $\mathbb{E}$ denotes expectation, and $\mathcal{C N}(\mathbf{m}, \mathbf{C})$ denotes a multivariate proper complex normal distribution with mean vector $\mathbf{m}$ and covariance matrix $\mathbf{C}$.

\section{SYSTEM MODEL}

We consider a multi-user, multi-antenna wideband downlink channel in which a base station (BS) equipped with $M$ antennas communicates with $K \leq M$ user equipments (UEs), each equipped with one antenna (see Fig. 1). Such a channel is also called a broadcast channel (BC) in the information theory literature. We use orthogonal frequency division multiplexing (OFDM) and thus the sampled received signal $y_{k, m, q} \in \mathbb{C}$ of the $k$-th user at time $m$ and subcarrier $q$ is mathematically described as

$$
y_{k, m, q}=\mathbf{h}_{k, m, q}^{T} \mathbf{x}_{m, q}+n_{k, m, q} \quad \text { for } \quad k=1, \ldots, K
$$

where $\mathbf{h}_{k, m, q} \in \mathbb{C}^{M}$ represents the $k$-th user channel response and $n_{k, m, q} \in \mathcal{C N}\left(0, \sigma^{2}\right)$ represents the circularly symmetric additive white Gaussian noise with zero mean and variance $\sigma^{2}$. The vector of transmit symbols $\mathbf{x}_{m, q} \in \mathbb{C}^{M}$ is a function of the multiple users' transmit symbols $\mathbf{x}_{k, m, q}$ with covariance matrix $\boldsymbol{\Sigma}_{k, m, q}=\mathbb{E}\left\{\mathbf{x}_{k, m, q} \mathbf{x}_{k, m, q}^{H}\right\}$. The sub-indices $m$ and $q$ always refer to the time and subcarrier indices, respectively.

The transmitter is subject to a power constraint per subcarrier, i.e., $\mathbf{x}_{m, q}^{H} \mathbf{x}_{m, q} \leq P$. The total transmit power is not 
dependent on the number of transmit antennas. Note that we always assume that we transmit to exactly $K=M$ users and we do not study the impact of user scheduling or power control. Further we assume that the noise power $\sigma^{2}=1$.

Equation (1) can also be written in matrix notation by defining $\mathbf{H}_{m, q}=\left[\mathbf{h}_{1, m, q} \ldots \mathbf{h}_{K, m, q}\right]^{T}$ and the vectors $\mathbf{y}_{m, q}$ and $\mathbf{n}_{m, q}$ accordingly:

$$
\mathbf{y}_{m, q}=\mathbf{H}_{m, q} \mathbf{x}_{m, q}+\mathbf{n}_{m, q} .
$$

We assume that each of the receivers has perfect and instantaneous knowledge of its own channel. Further we assume a zero-delay error-free feedback channel and denote the channel matrix fed back at the transmitter with $\hat{\mathbf{H}}$. We consider two cases for the feedback: ( $i$ ) full feedback, i.e., the bandwidth is large enough to feed back the full channel estimate and (ii) limited feedback with a resolution of $B$ bits for each subcarrier $q$ and time $m$. For notation convenience, we drop the time and subcarrier indices $m$ and $q$ when their dependence is not needed.

\section{Sum Rates of Multi-USER MiMO ChanNELS}

In this section we review the capacity of multi-user MIMO channels (Subsection III-A) as well as the sum rate of linear precoding schemes (Subsection III-B). For comparison we will also review the sum rate of a multiuser system employing single-user multiple-input single-output (SU-MISO) TDMA in Subsection III-C.

\section{A. Capacity}

From the results in $[6,25]$, the sum capacity of the MUMIMO downlink channel can be expressed by the following maximization:

$$
\begin{aligned}
& \mathcal{C}_{\mathrm{BC}}(\mathbf{H}, P)= \\
& \max _{\boldsymbol{\Sigma}_{k} \geq 0, \sum_{k=1}^{K} \operatorname{tr}\left(\boldsymbol{\Sigma}_{k}\right) \leq P} \sum_{k=1}^{K} \log _{2} \frac{1+\mathbf{h}_{k}^{H}\left(\sum_{j=1}^{K} \boldsymbol{\Sigma}_{j}\right) \mathbf{h}_{k}}{1+\mathbf{h}_{k}^{H}\left(\sum_{j \neq k} \boldsymbol{\Sigma}_{j}\right) \mathbf{h}_{k}},
\end{aligned}
$$

where the maximization is over the set of all positive semidefinite transmit covariance matrices $\boldsymbol{\Sigma}_{k}, k=1, \ldots, K$. The objective function of the maximization in (3) is a non-convex function of the covariance matrices, making it very difficult to deal with. Fortunately, due to the duality of the BC and the multiple access channel (MAC) [16], the sum rate capacity of the MIMO BC is equal to the sum rate capacity of the dual MAC with power constraint $P$

$$
\begin{aligned}
& \mathcal{C}_{\mathrm{BC}}(\mathbf{H}, P)=\mathcal{C}_{\mathrm{MAC}}(\mathbf{H}, P)= \\
& \max _{\mathbf{Q}_{k} \geq 0, \sum_{k=1}^{K} \operatorname{tr}\left(\mathbf{Q}_{k}\right) \leq P} \log _{2}\left(1+\sum_{k=1}^{K} \mathbf{h}_{k}^{H} \mathbf{Q}_{k} \mathbf{h}_{k}\right),
\end{aligned}
$$

where each of the matrices $\mathbf{Q}_{i}$ is a positive semidefinite covariance matrix. Since (4) involves the maximization of a convex function, efficient numerical algorithms exist. In this paper, we use the specialized algorithm developed in [26] to calculate $\mathcal{C}_{\mathrm{BC}}(\mathbf{H}, P)$.
It has been shown [7] that the sum rate capacity given in Equation (4) is actually achieved by using DPC. However, DPC is complex to implement in practical systems and thus we also study linear precoding schemes in the next section.

\section{B. Linear Precoding}

Let $s_{k} \in \mathbb{C}$ denote the $k$-th user data symbol. Under linear precoding, the transmitter multiplies the data symbols $s_{k}$ by the precoding vectors $\mathbf{w}_{k} \in \mathbb{C}^{M}$ and combines them to the transmit symbol $\mathbf{x}$, i. e., $\mathbf{x}=\sum_{k=1}^{K} \mathbf{w}_{k} s_{k}$. In order to fulfill the transmit power constraint, the transmitter further normalizes $\mathbf{x}$, such that $\mathbf{x}^{H} \mathbf{x}=P$. From (1) the resulting received signal vector for user $k$ is then given by

$$
y_{k}=\mathbf{h}_{k}^{T} \mathbf{w}_{k} s_{k}+\sum_{j \neq k} \mathbf{h}_{k}^{T} \mathbf{w}_{j} s_{j}+n_{k},
$$

where the first term is the desired signal, the second term represents the multi-user interference, and the last term the noise. The signal to noise plus interference ratio (SINR) at each user $k$ is thus given by

$$
\mathrm{SINR}_{k}=\frac{\left|\mathbf{h}_{k}^{T} \mathbf{w}_{k}\right|^{2}}{\sum_{j \neq k}\left|\mathbf{h}_{k}^{T} \mathbf{w}_{j}\right|^{2}+\sigma^{2}} .
$$

If the user codes are drawn from an i.i.d. Gaussian distribution, the sum rate of linear precoding is thus given by

$$
\mathcal{R}_{\mathrm{BC}}(\mathbf{H}, P)=\sum_{k=1}^{K} \log _{2}\left(1+\mathrm{SINR}_{k}\right) .
$$

In this paper we use a regularized channel inversion with equal power allocation based on the feedback channel to design the precoding vectors $\mathbf{w}_{k}$ [8]. The regularized channel inverse is given by

$$
\mathbf{W}=\hat{\mathbf{H}}^{H}\left(\hat{\mathbf{H}} \hat{\mathbf{H}}^{H}+\beta \mathbf{I}\right)^{-1},
$$

where $\hat{\mathbf{H}}=\left[\hat{\mathbf{h}}_{1}, \ldots, \hat{\mathbf{h}}_{K}\right]^{T}$ is the fed back channel matrix and $\beta$ is the regularization factor. The precoding vectors $\mathbf{w}_{k}$ are finally given by the columns of $\mathbf{W}$.

The above scheme is often referred to as MMSE precoding with equal power allocation due to the analogy with MMSE beamforming weight design criterion if the noise is spatially white. If $\beta=0$, Equation (8) reduces to the $\mathrm{ZF}$ precoder. However, when the channel matrix is ill-conditioned, at least one of the singular values of $\left(\hat{\mathbf{H}} \hat{\mathbf{H}}^{H}\right)^{-1}$ is very large, resulting in a very low signal to noise ratio (SNR) at the receivers.

A non-zero $\beta$ value on the other hand allows for a certain amount of multi-user interference. The amount of interference is determined by $\beta>0$ and an optimal tradeoff between the condition of the channel matrix inverse and the amount of crosstalk ought to be found. In practice, the regularization factor is commonly chosen as $\beta=M \sigma^{2} / P$ motivated by the results in [8] that show that it approximately maximizes the SINR at each receiver, and leads to linear capacity growth with $M$. The performance of MMSE is certainly significantly better at low SNR and converges to that of $\mathrm{ZF}$ precoding at high SNR. However, MMSE does not provide parallel and orthogonal channels and thus power allocation techniques cannot be performed in a straightforward manner. 


\section{Time Division Multiple Access}

In a TDMA system, the BS only serves one user at a time. We analyze the case when full CSIT and with no CSIT. In the case of full CSIT, the capacity of a particular user $k$ is given by

$$
\mathcal{C}_{\text {SU-CSIT }}\left(\mathbf{h}_{k}, P\right)=\log _{2}\left(1+\frac{P}{\sigma^{2}}\left\|\mathbf{h}_{k}\right\|^{2}\right) .
$$

The capacity is achieved by transmit maximum ratio combining [27].

If full CSIT is available the sum rate of the system can be optimized by transmitting to the user with the largest single-user capacity only, exploiting multi-user diversity [28]. However, with multiple transmit antennas and a small number of users (which is the case considered in this paper) the gains of multi-user diversity are reduced. Therefore, we assume that all users are allocated an equal amount of time (round robin scheduling). This also allows a more fair comparison to the linear precoding schemes considered in the previous section. The sum rate of the system is thus given by

$$
\mathcal{C}_{\text {TDMA-CSIT }}(\mathbf{H}, P)=\sum_{k=1}^{K} \frac{1}{K} \mathcal{C}_{\text {SU-CSIT }}\left(\mathbf{h}_{k}, P\right) .
$$

When no CSIT is available, the capacity of a particular user $k$ is given by

$$
\mathcal{C}_{\text {SU-noCSIT }}\left(\mathbf{h}_{k}, P\right)=\log _{2}\left(1+\frac{P}{\sigma^{2} M}\left\|\mathbf{h}_{k}\right\|^{2}\right) .
$$

Again, we assume that multiple users are served using a round robin scheduler and thus the sum rate $\mathcal{C}_{\text {TDMA-noCSIT }}(\mathbf{H}, P)$ can be defined similar to (10).

\section{Obtaining CSIT through Limited FeEdback}

The linear precoding schemes described in the last section require CSIT in the form of the matrix $\hat{\mathbf{H}}$. When a feedback channel with a limited bandwidth (as described in Section II) is available, channel vector quantization can be used to feed back a quantized version of the channel, providing partial CSIT. Such a scheme has also been proposed for LTE [4] and is outlined in Section IV-A.

By exploiting time-correlation in the channel, the actual feedback rate can be reduced. Recently, it has been pointed out in [17] that the actual required feedback rate is given by the CSI source rate. This measure is introduced in Subsection IV-B.

\section{A. Channel Vector Quantization}

For each subcarrier $q$ and every time index $m$, the UE $k$ selects a quantization vector with index $I_{k, m, q}$ from a codebook $\mathcal{C}=\left\{\mathbf{c}_{1}, \ldots, \mathbf{c}_{C}\right\}$ of size $C=2^{B}$, such that the angle between the actual channel $\mathbf{h}_{k, m, q}$ and the codeword $\mathbf{c}_{I_{k, m, q}}$ is minimized. This is equivalent to writing

$$
I_{k, m, q}=\underset{i=1, \ldots, C}{\operatorname{argmax}}\left|\mathbf{c}_{i}^{H} \mathbf{h}_{k, m, q}\right| .
$$

For every subcarrier, the UE then feeds back the index $I_{k, m, q}$ along with a channel quality information (CQI). In this paper we use the channel vector norm $\left\|\mathbf{h}_{k, m, q}\right\|$ as CQI. Note that this choice of CQI is not suitable for multi-user scheduling, since it does not take the multi-user interference and the quantization error into account. However, in this paper we are only interested in the precoder design and do not consider scheduling. Moreover, we assume that the channel vector norm is not quantized, since we are only interested in the ability of the codebook to capture the spatial properties of the channel.

The transmitter, which also knows the codebook, can then reconstruct the channel by a simple lookup table: $\hat{\mathbf{h}}_{k, m, q}=$ $\mathbf{c}_{I_{k, m, q}}\left\|\mathbf{h}_{k, m, q}\right\|$. The codebook $\mathcal{C}$ is designed off-line and there are several well-known possibilities. In this paper we consider a Grassmannian codebook, a Fourier codebook, a random codebook and a correlated random codebook.

1) Grassmannian Codebook: The Grassmannian codebook derives its name from the Grassmannian line packing problem, which is defined as follows [29]: how should $C$ onedimensional subspaces of the $M$-dimensional (complex) Euclidean space be arranged so that they are as far apart as possible? This problem is equivalent to finding the optimal quantization vectors of a source with uniform distribution on the (complex) $M$-dimensional unit sphere. Therefore the resulting codebook is optimal if the elements of the channel vectors $\mathbf{h}_{k}$ are i.i.d. complex Gaussian distributed [30]. In this work we use the Grassmannian codebook available for download at [31].

2) Fourier Codebook: The Fourier codebook is obtained by defining $\mathbf{c}_{i}$ as the top $M$ rows of the $i$-th column of the discrete Fourier transform (DFT) matrix of size $C$, i. e.,

$$
\mathbf{c}_{i}=\frac{1}{\sqrt{M}}\left[1, e^{-2 \pi j i / C}, \ldots, e^{-2 \pi j i(M-1) / C}\right]^{T} .
$$

Each entry of the codebook can be interpreted as a beamforming vector of a linear antenna array with one fixed beam. This codebook is therefore well suited for line of sight (LOS) channels with linear antenna arrays.

The Fourier codebook index calculation (12) can be implemented efficiently (in terms of memory and computation) by means of an inverse fast Fourier transform (IFFT). Also, the codebook does not need to be stored at the transmitter, as it can be easily reconstructed [32].

3) Random Codebook: The quantization vectors of the random codebook are constructed by drawing $\mathbf{c}_{i}$ randomly from an i.i.d. complex Gaussian distribution on the $M$-dimensional unit sphere, i. e., $\mathbf{c}_{i} \in \mathcal{C N}\left(\mathbf{0}_{M}, \mathbf{I}_{M}\right)$ and subsequent normalization, i. e., $\left\|\mathbf{c}_{i}\right\|=1$.

4) Correlated Random Codebook: The quantization vectors of the correlated random codebook are drawn from complex Gaussian distribution on the $M$-dimensional unit sphere, whose covariance matrix matches the transmit correlation matrix of the channel, i. e., $\mathbf{c}_{i} \in \mathcal{C N}\left(\mathbf{0}_{M}, \mathbf{R}_{\mathrm{Tx}}^{(k)}\right)$ and $\left\|\mathbf{c}_{i}\right\|=1$. The transmit correlation matrix is defined as

$$
\mathbf{R}_{\mathrm{Tx}}^{(k)}=\mathbb{E}\left\{\mathbf{h}_{k} \mathbf{h}_{k}^{H}\right\} .
$$

The application scenario of correlated codebooks considered in this paper is that different BSs or even different sectors of a BS employ codebooks that are adapted to their environment. The transmit correlation matrix should thus be estimated over 


\begin{tabular}{|c|c|c|}
\hline Parameter & Meas. 1-3 & Meas. 4 \\
\hline Center Frequency & $1917.6 \mathrm{MHz}$ & $1917.6 \mathrm{MHz}$ \\
Useful Bandwidth & $4.8 \mathrm{MHz}$ & $4.0625 \mathrm{MHz}$ \\
BS Transmit Power & $30 \mathrm{dBm}$ & $30 \mathrm{dBm}$ \\
Number of Antennas at BS $(M)$ & 4 & 2 \\
Number of UE $(K)$ & 4 & 2 \\
Number of Antennas at UE $(N)$ & 1 & 1 \\
Number of Subcarriers $(Q)$ & 40 & 80 \\
\hline
\end{tabular}

TABLE I

PARAMETERS OF THE EURECOM MIMO OPENAIR SOUNDER FOR THE FOUR MEASUREMENT CAMPAIGNS.

a wide frequency range and several locations. In this paper we estimate $\mathbf{R}_{\mathrm{Tx}}^{(k)}$ from the measurements by taking the mean of $\mathbf{h}_{k} \mathbf{h}_{k}^{H}$ over all frequencies $q$ and all frames $m$ in one measurement.

For both the random and the correlated random codebook we assume that each user has a different and independently generated quantization codebook as in [11].

\section{B. Feedback Rate}

In order to evaluate the intrinsic rate of information brought by the measured channel, we follow [17] and model the time variation of $I_{k, m, q}$ as a finite-state Markov chain of order 1 with $C$ states (see Fig. 8 for an example). Let $\mathbf{P}^{(k, q)}$ be the transition probability matrix with elements $P_{l, n}^{(k, q)}=$ $\operatorname{Pr}\left(I_{k, m, q}=l \mid I_{k, m-1, q}=n\right)$. Also define the stationary probability vector $\pi^{(k, q)}$ with elements $\pi_{l}^{(k, q)}=\operatorname{Pr}\left(I_{k, m, q}=l\right)$. $\mathbf{P}^{(k, q)}$ and $\pi^{(k, q)}$ are estimated from the measurements.

We now use Proposition 1 of [17] to calculate the normalized CSI source bit rate per user and per subcarrier

$$
\mathcal{R}_{\mathrm{CSI}}(k, q)=B \sum_{l=1}^{C} \pi_{l}^{(k, q)}\left(1-P_{l, l}^{(k, q)}\right) .
$$

When evaluating the measurements we take the mean over all frequencies $q$ like we do for the capacity evaluations. Further, in the multi-user case we are interested in the sum rate and thus sum over all users $k$

$$
\mathcal{R}_{\mathrm{CSI}}=\frac{1}{Q} \sum_{k=1}^{K} \sum_{q=1}^{Q} \mathcal{R}_{\mathrm{CSI}}(k, q) .
$$

\section{Measurements And Results}

In this section we present results using real channel measurement data. We first describe the measurement scenarios in Subsection V-A and the normalization of the recorded channel matrices in V-B. Finally, in Subsection V-C we apply the metrics for the MU-MIMO sum rate from Section III and the feedback rate from Section IV directly to the recorded and normalized channel matrices.

\section{A. Measurement Description}

The measurements were conducted using the Eurecom MIMO OpenAir Sounder (EMOS) $[14,18,33]$ in the vicinity of the Eurecom institute in Sophia-Antipolis, France. The scenario is characterized by a semi-urban hilly terrain, composed by short buildings and vegetation. Fig. 2 shows

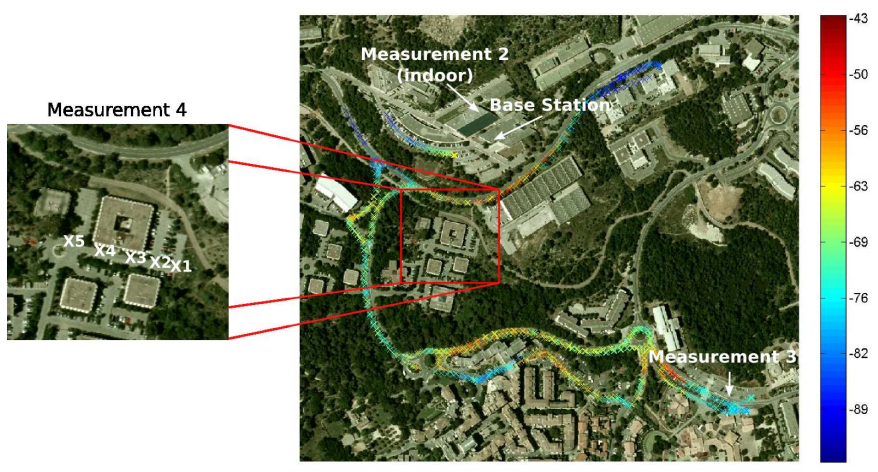

Fig. 2. Map of the measurement scenario. In measurement 1 the users were driving in cars along the indicated routes (the colors show the received signal strength in $\mathrm{dBm}$ along the routes). Measurements 2-4 are indicated on the map.

a map of the environment. The BS is located at the roof of Eurecom's southmost building. The antenna is directed towards Garbejaire, a small nearby village. The colors indicate the received signal strength along the measurement routes. The measurement parameters are summarized in Table I. A more detailed description of the EMOS can be found in the Appendix.

In the first three measurements, we use all four transmit antennas (arranged in 2 cross-polarized pairs) and four users with one antenna each. In the first measurement, the UEs were placed inside standard passenger cars which were being driven along the routes shown in Fig. 2. The cars had no fixed routes and thus the distance between them was changing. In the second measurement, the users were indoors in the same room, walking around slowly. In the third measurement, the users were parked close together in a parking lot.

In the fourth measurement we use only two co-polarized antennas at the transmitter and two users with one antenna each. The first user is always at position $x_{1}$ and the second user is at position $x_{i}, i=1, \ldots, 5$. Positions $x_{1}, x_{2}$, and $x_{5}$ are LOS while positions $x_{3}$ and $x_{4}$ are behind an office building. During the measurements the users were moving only within a few wavelengths in order to get a sufficient number of samples for the evaluation of the statistics of the small scale fading.

\section{B. Normalization}

In order to control the average SNR at the UEs, we have to re-normalize the recorded MIMO channel matrices. One measurement results in the set of MIMO channel matrices

$$
\begin{aligned}
\left\{\mathbf{H}_{k, m, q} \in \mathbb{C}^{N \times M}, k=0, \ldots, K-1,\right. & \\
m & \left.=0, \ldots, N_{\mathrm{F}}-1, q=0, \ldots, Q-1\right\},
\end{aligned}
$$

where $k$ denotes the user index, $m$ the snapshot index, and $q$ the frequency (or subcarrier) index. $N, M$, and $K$ are the number of receive antennas, number of transmit antennas and number of users respectively. $N_{\mathrm{F}}$ is the total number of snapshots per measurement after removing erroneous frames (on average $N_{\mathrm{F}} \approx 18.000$, corresponding to approx. $50 \mathrm{sec}$ ). The total number of channel estimates in the frequency domain is given by $Q=160 / M$, since there are 160 subcarriers 


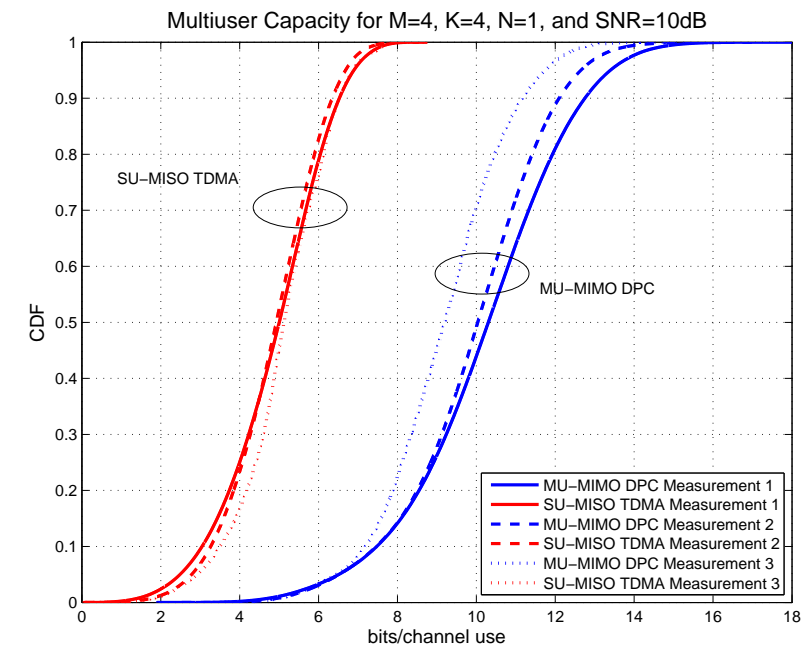

Fig. 3. CDF of the sum rate of SU-MISO TDMA compared to MU-MIMO with DPC for measurements 1-3. The average SNR is fixed to $10 \mathrm{~dB}$ for each user.

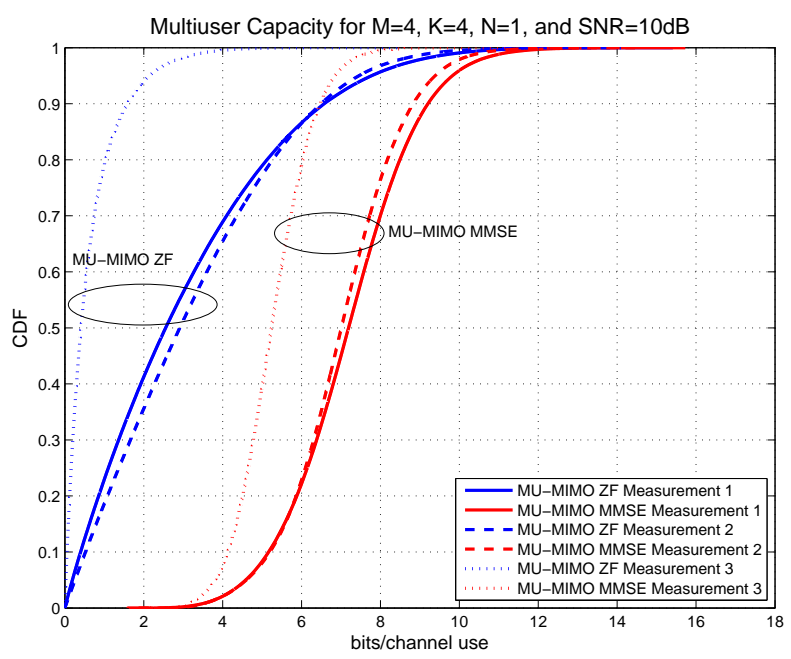

Fig. 4. CDF of the sum rate of MU-MIMO with ZF and MMSE precoding for measurements 1-3. The average SNR is fixed to $10 \mathrm{~dB}$ for each user.

in total and the pilots are multiplexed over the $M$ transmit antennas. The MIMO matrices are normalized by

$$
\mathbf{H}_{k, m, q}^{\prime}=\mathbf{H}_{k, m, q} \sqrt{\frac{N N_{\mathrm{F}} Q}{\sum_{m, q}\left\|\mathbf{H}_{k, m, q}\right\|_{F}^{2}}}
$$

such that $\mathbb{E}\left\{\left\|\mathbf{H}_{k}^{\prime}\right\|_{F}^{2}\right\}=N$. Since the noise variance $\sigma^{2}=1$, the average SNR at each UE $k$ is thus $\frac{N P}{K}$.

\section{Results}

1) Comparing different scenarios: Firstly, we compare the performance of MU-MIMO using DPC, ZF precoding, and MMSE precoding as well as SU-MISO TDMA based on the empirical cumulative density function (CDF) of the sum rate (Equations (4), (7), and (10)). We assume an average SNR at the users of $10 \mathrm{~dB}$, which corresponds to the average SNR at the cell edge. The results are plotted in Figures 3 and 4 for measurements 1-3. Secondly, we compare the mean MUMIMO sum rate for all the above mentioned schemes with

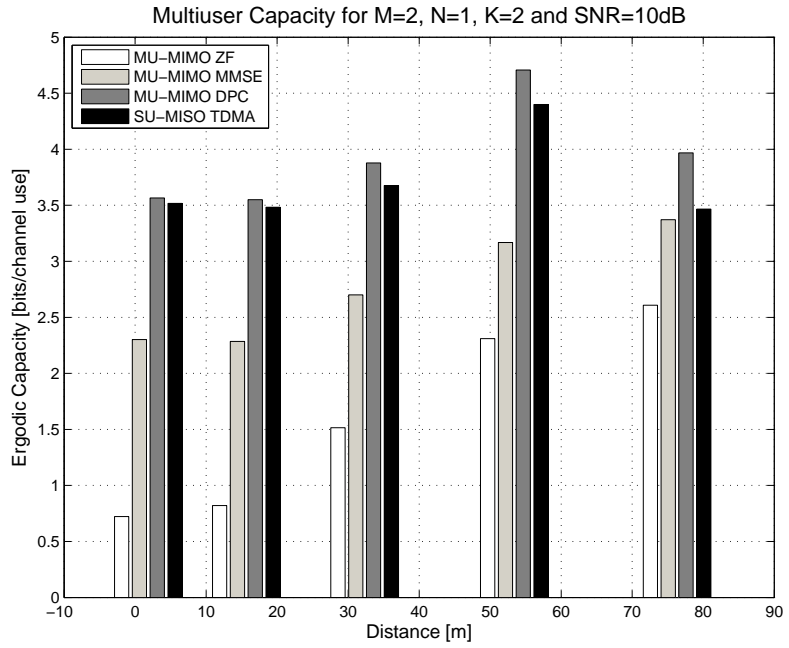

Fig. 5. Comparison of mean MU-MIMO sum rate for DPC and SU-MISO TDMA, as well as ZF and MMSE precoding with respect to inter-user distance for measurement 4 . The average SNR is fixed to $10 \mathrm{~dB}$ for each user.

respect to the inter-user distance. The results are plotted in Fig. 5 for measurement 4 .

It can be seen from Fig. 3 that MU-MIMO DPC as well as SU-MISO TDMA do not show a very high variability with respect to the different measurements. However, the linear MU-MIMO precoding schemes (see Fig. 4) are very sensitive to the channel conditions. Especially the performance of the ZF precoder drops significantly in the outdoor scenario where the users are close together. In the indoor scenario and the other outdoor scenario where all users are well separated, the performance of the linear MU-MIMO schemes is comparable.

The effect of the inter-user distance on the capacity of the different schemes can be observed more closely in Fig. 5. It can be seen that there is a clear relationship between the distance and the capacity of linear precoding schemes: the further apart the users are, the higher the capacity. In fact, for inter-user distances up to 55m, the SU-MISO TDMA scheme always performs better than the linear MU-MIMO schemes. Only in the last measurement at $75 \mathrm{~m}$, the MMSE precoder shows a slightly better performance than the TDMA scheme.

The poor performance of linear precoders in scenarios with a small inter-user distance can be explained by looking at the channel correlation matrix of the different scenarios $[15$, 34]. When the channel is strongly correlated it means that the channel matrix is ill-conditioned. Thus at least one of the singular values of $\left(\mathbf{H H}^{H}\right)^{-1}$ is very large, resulting in a very low SNR at the receivers, when ZF precoding is used. The MMSE precoder can alleviate this problem, but still suffers from the high correlation at the transmitter.

2) Comparing Different Codebooks: Fig. 6 and 7 compare the CDF of the MU-MIMO sum rate using MMSE precoding based on quantized feedback using different codebooks of size 64 (6 bit) and size 4096 (12 bit) respectively. We also plot the cases with perfect feedback (cf. Equation (7)) and no feedback (cf. Equation (11)) as lower and upper bounds for comparison. Measurement 1 are used for both plots.

It can be seen that the performance of MU-MIMO with 


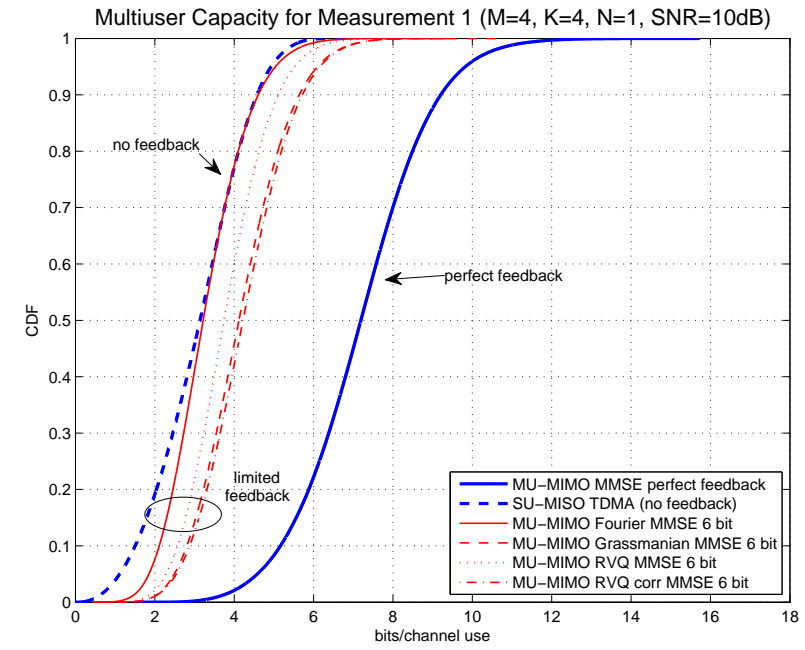

Fig. 6. CDF of the sum rate of MU-MIMO with MMSE precoding with different feedback schemes for measurement 1 . The average SNR is fixed to $10 \mathrm{~dB}$ for each user. We compare the performance under several codebooks of size 64 (6 bit) with the perfect feedback case as well as to the case with no feedback, which is equivalent to SU-MISO TDMA.

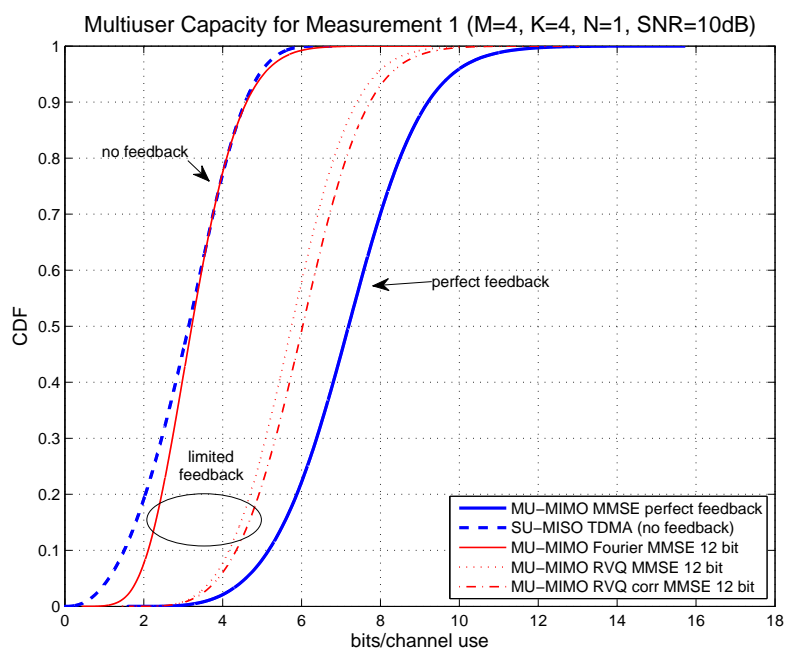

Fig. 7. CDF of the sum rate of MU-MIMO with MMSE precoding with different feedback schemes for measurement 1 . The average SNR is fixed to $10 \mathrm{~dB}$ for each user. We compare the performance under several codebooks of size 4096 (12 bit) with the perfect feedback case as well as to the case with no feedback, which is equivalent to SU-MISO TDMA.

MMSE precoding depends strongly on the chosen codebook. For the evaluated outdoor channel, the Fourier codebook exhibits the worst performance, being only slightly better than a SU-MISO TDMA scheme with no feedback at all. Further, its performance does not increase with the number of feedback bits. The correlated random codebook performs better than the Fourier codebook. The random codebook and the Grassmannian codebook perform best. However, for 6 bits of feedback the gap to the perfect feedback case is still significant ( $3 \mathrm{bits} / \mathrm{sec} / \mathrm{Hz}$ at $50 \%$ outage rate). Doubling the number of feedback bits to 12 reduces the gap to 1.2 $\mathrm{bits} / \mathrm{sec} / \mathrm{Hz}$ (at $50 \%$ outage rate), which is comparable to the theoretical results achieved in [11]. The gain to the SU-MISO

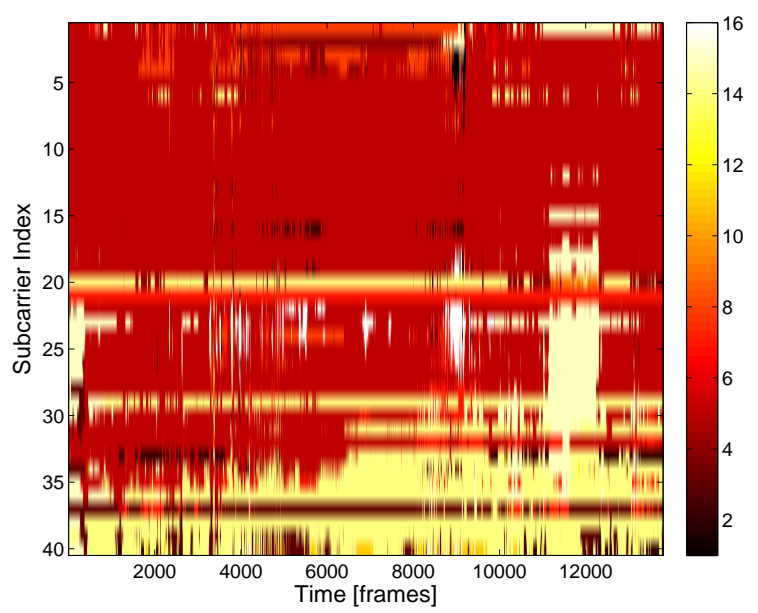

Fig. 8. Calculated codebook indices $I_{1, m, q}$ using a 4 bit Grassmannian codebook on measurement 1 . The different shades of gray correspond to different codebook indices. It can be seen that for some subcarriers the volatility of the codebook indices is quite high (resulting in a higher feedback rate) while for other subcarriers it is rather low (resulting in a low feedback rate).

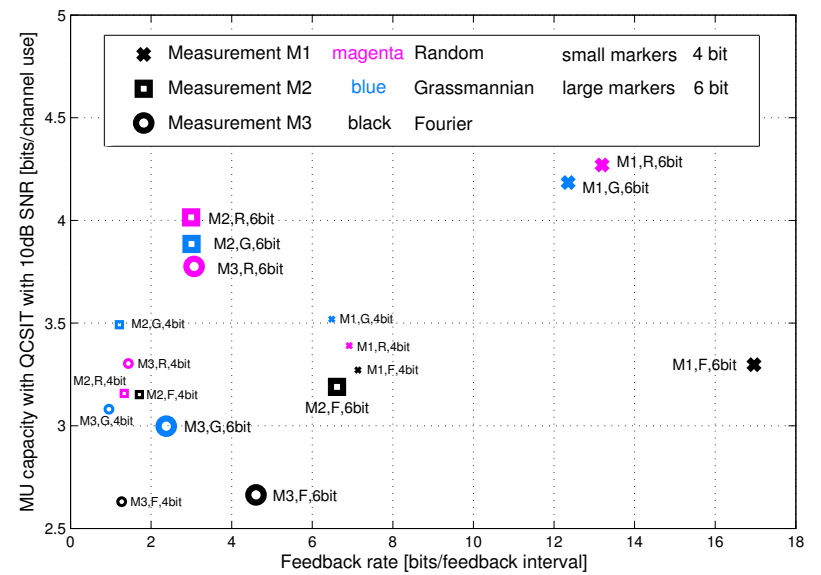

Fig. 9. CSI source rate of the output of the channel quantizer $\mathcal{R}_{\mathrm{CSI}}$ vs. MUMIMO sum rate $\mathcal{R}_{\mathrm{MMSE}}$. Each point in the graph represents the result for one measurement, one codebook and one codebook size. This figure allows us to compare different codebooks with respect to their ability to capture the spatial and temporal properties of the channel. The used measurements, codebooks and codebook sizes are given in the legend as well in the figure next to the data point.

TDMA system is $2.9 \mathrm{bits} / \mathrm{sec} / \mathrm{Hz}$ (at $50 \%$ outage rate).

The poor performance of the Fourier codebook can be explained by the fact that $(i)$ the BS does not use a linear antenna array and (ii) the investigated channel does not have a LOS component. Further, the fact that the performance of the Fourier codebook does not increase with the codebook size can be explained by looking at the maximum cross-correlation between codebook entries, $f(C)=\max _{\mathbf{c}_{i}, \mathbf{c}_{j} \in C, i \neq j}\left|\mathbf{c}_{i}^{H} \mathbf{c}_{j}\right|$. In the case of a Fourier codebook, $f(C)$ will converge to one as the codebook size increases (just choose two neighboring codebook entries $\mathbf{c}_{i}$ and $\mathbf{c}_{i+1}$ ). For the random codebook on the other hand $f(C)$ will converge to zero, since any two codewords are uncorrelated with probability one [35]. 
3) Feedback Rate vs Capacity: For illustration purposes we plot the quantized channel indices $I_{k, m, q}(12)$ for measurement 1 for user $k=1$ in Fig. 8. It can be seen that for some frequencies $q$ the channel remains quite constant whereas for other frequencies it varies more.

In Fig. 9 we plot the CSI source rate $\mathcal{R}_{\mathrm{CSI}}(16)$ vs. the MU-MIMO sum rate $\mathcal{R}_{\text {MMSE }}$ (7) for measurements $1-3$, three different codebooks and two codebook sizes. This figure gives an indication of how well the codebook is able to exploit the temporal correlation in the channel (low feedback rate) with respect to the gain in capacity it brings. Points further to the top left of the figure are the best (low feedback rate while having high capacity).

It can be seen that the results are quite different for different codebooks and for the different measurements. Looking at the Fourier codebook (black markers), it can be seen that this codebook requires the largest feedback rate while providing the lowest sum rate. Increasing the number of feedback bit just increases the feedback rate and not the capacity. Considering the Grassmannian codebook (blue markers) on the other hand, it can be seen that this codebook requires much less feedback than the Fourier codebook for the same codebook size while having a larger capacity. Further, increasing the codebook size increases the capacity significantly.

The random codebook has similar properties as the Grassmannian codebook. A 4 bit random codebook offers a lower capacity than a 4 bit Grassmannian codebook, whereas a 6 bit random codebook offers higher capacity than a 6 bit Grassmannian codebook. Also interesting to note is that the higher mobility measurements require more feedback than the low mobility measurements, but at the same time have a higher capacity.

\section{CONCLUSIONS}

We have presented an extensive evaluation of different MUMIMO schemes with perfect and limited feedback in various channel conditions. The data was acquired using Eurecom's MU-MIMO channel sounder EMOS.

From the results we can derive two important criteria that need to be considered when designing MU-MIMO schemes. Firstly, spatial separation of users has a very strong impact on the performance of linear precoding schemes. In particular, the performance of a ZF precoder drops significantly in outdoor scenarios, when the users are close together. Therefore it is necessary to design proper scheduling algorithms that select users with different spatial signatures.

Secondly, the performance of limited feedback MU-MIMO schemes crucially depends on the codebook. It was seen that the performance of the Fourier codebook is hardly better than that of a SU-MISO TDMA scheme with no feedback at all, even for a high number of feedback bits. Further this codebook does not allow any feedback reduction in time-correlated channels. We thus conclude that the Fourier codebook is not able to capture the spatial properties of the measured outdoor wideband channel appropriately. The performance of the random codebook, the Grassmannian codebook and especially the correlated random codebook increases with the number

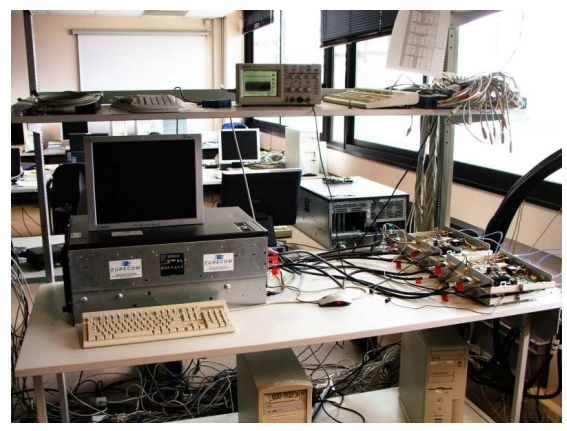

(a) BS with PLATON boards

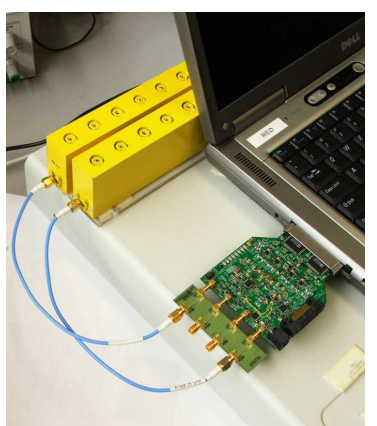

(c) UE with CardBus MIMO board

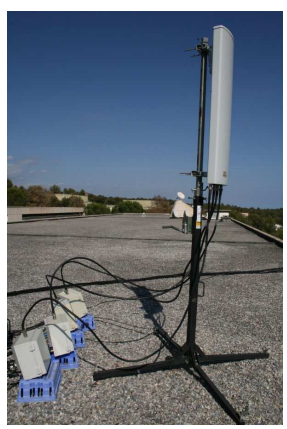

(b) Power amplifiers and Powerwave antenna
Fig. 10. EMOS base station and user terminals [18].

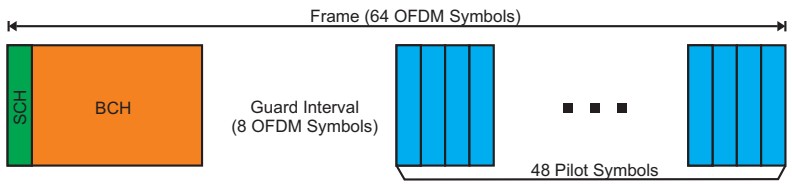

Fig. 11. Frame structure of the OFDM Sounding Sequence. The frame consists of a synchronization channel, $(\mathrm{SCH})$, a broadcast channel $(\mathrm{BCH})$, and several pilot symbols used for channel estimation.

of feedback bits. Also, these codebooks allow for a feedback reduction in time-correlated channels. Thus, these codebooks are able to represent the channel more appropriately.

It can be concluded that the codebook design for MUMIMO systems remains a hot topic. The tools and the analysis presented in this paper allow to carefully evaluate different codebooks and to choose the codebook that provides a good trade-off between feedback and downlink rate.

\section{APPENDIX}

\section{THE EURECOM MIMO OPEnAIR SOUNDER}

This Appendix describes the Eurecom MIMO OpenAir Sounder (EMOS). We start by giving an overview of the hardware architecture, followed by a description of the sounding signal and the synchronization procedure. Last, but not least we explain how we perform high-accuracy MIMO channel estimates. [14, 34, 35].

\section{A. Hardware Description}

The EMOS is based on the OpenAirInterface ${ }^{1}$ hardware/ software development platform at Eurecom. The platform

\footnotetext{
${ }^{1}$ http://www.openairinterface.org
} 
consists of a BS that continuously sends a signaling frame, and one or more UEs that receive the frames to estimate the channel. The BS consists of a workstation with four baseband data acquisition cards, which are connected to four radio-frequency (RF) boards (called PLATON, see Fig. 10(a)). The RF signals are amplified and transmitted by a Powerwave $3 \mathrm{G}$ broadband antenna composed of four elements which are arranged in two cross-polarized pairs (part no. 7760.00, see Fig. 10(b)). The UEs consist of a laptop computer with Eurecom's dual-RF data acquisition card (called CardBus MIMO, see Fig. 10(c)) and two clip-on 3G Panorama Antennas (part no. TCLIP-DE3G, see Fig. 10(d)). Both equipments operate at $1.900-1.920 \mathrm{GHz}$ with $5 \mathrm{MHz}$ channels ${ }^{2}$. The platform is designed for a full software-radio implementation, in the sense that all protocol layers run on the host PCs under the control of the real-time application interface ${ }^{3}$ (RTAI), which is an extension to the Linux operating system.

\section{B. Sounding Signal}

The EMOS is using an orthogonal frequency division multiplexing (OFDM) modulated sounding sequence with 256 subcarriers (out of which 160 are non-zero) and a cyclic prefix length of 64. One transmit frame is 64 OFDM symbols (2.667 ms) long and consists of a synchronization symbol (SCH), a broadcast data channel $(\mathrm{BCH})$ comprising 7 OFDM symbols, a guard interval, and 48 pilot symbols used for channel estimation (see Fig. 11). The pilot symbols are taken from a pseudo-random quadrature phase-shift keying (QPSK) sequence defined in the frequency domain. The subcarriers of the pilot symbols are multiplexed over the four transmit antennas to ensure orthogonality in the spatial domain. We can therefore obtain one full MIMO channel estimate for one group of $M$ subcarriers. The BCH uses QPSK modulation and rate $1 / 2$ convolutional code and contains (among other information) the frame number of the transmitted frame that is used for synchronization among the UEs.

\section{Synchronization}

Transmitter and receiver must be synchronized in order to conduct usefull measurements. Synchronization is taking place at three different levels, which are described below.

1) Initial Synchronization: Initial synchronization is performed using a sliding window correlator on the $\mathrm{SCH}$ symbol in the frequency domain. After successfull detection of the $\mathrm{SCH}$, a channel estimate is performed on the $\mathrm{SCH}$. This channel estimate is used for coherent detection of the $\mathrm{BCH}$ with a Viterbi decoder. Synchronization is declared only if the $\mathrm{BCH}$ can be detected successfully, i.e., the cyclic redundancy check (CRC) is positive.

2) Synchronization Tracking: Due to the drifts of the sampling clocks of transmitter and receiver, as well as the movement of the user, the synchronization needs to be adjusted constantly. This is done by tracking the peak of the channel

\footnotetext{
${ }^{2}$ Eurecom has a frequency allocation for experimentation around its premises.

${ }^{3}$ http://www.rtai.org
}

estimate of the $\mathrm{SCH}$ in the time domain. To avoid jitter, the peak position is passed through a low-pass filter. If the peak position drifts from the target position by more than 5 samples, the timing offset of the hardware is increased (decreased) by one sample.

The receiver also continues to decode the $\mathrm{BCH}$. If the $\mathrm{BCH}$ cannot be detected successfully for 100 consecutive frames or more, the receiver declares itself out of sync and the initial synchronization procedure is stared again. For successful decoding of the $\mathrm{BCH}$, a SNR of approximately $10 \mathrm{~dB}$ or more is required.

3) Multi-user Synchronization: In order to conduct multiuser measurements, all the UEs need to be frame-synchronized to the BS. This is important for (i) synchronized start and stop of the data acquisition and (ii) for the proper alignment of the measurement data from multiple users in the post processing. Multi-user synchronization is achieved by using the frame number encoded in the $\mathrm{BCH}$. This frame number is also stored along with the measured channel at the UEs for post processing.

\section{EMOS Channel Estimation Procedure}

Once the receiver is fully synchronized to the transmitter, the EMOS channel estimation procedure is started. Note that this procedure uses all the 48 pilot symbols of a frame (cf. Fig. 11) and thus provides a more accurate channel estimate than the one based on the SCH symbol at the beginning of the frame, which is only used for synchronization and coherent decoding of the $\mathrm{BCH}$.

The EMOS channel estimation procedure consists of two steps. Firstly, the pilot symbols are derotated with respect to the first pilot symbol to reduce the phase-shift noise generated by the CardBus MIMO card. Secondly, the pilot symbols are averaged to increase the measurement SNR. The channel is then estimated in the frequency domain by multiplication of the derotated and averaged symbols with the complex conjugate of the pilot symbol. The estimated MIMO channel is finally stored to disk. For a more detailed description of the synchronization and channel estimation procedure see [18,33].

\section{ACKNOWLEDGMENTS}

The authors would like to thank their colleagues at Eurecom and G. Attard who helped to carry out the measurements for this paper. The authors would also like to thank the anonymous reviewers for their comments, which helped to improve the paper.

\section{REFERENCES}

[1] G. J. Foschini and M. J. Gans, "On limits of wireless communications in a fading environment when using multiple antennas," Wireless Personal Communications, vol. 6, no. 3, pp. 311-335, 1998.

[2] E. Telatar, "Capacity of multi-antenna Gaussian channels," European Transactions on Telecommunications, vol. 10, pp. 585-595, 1999.

[3] D. Gesbert, M. Shafi, D.-S. Shiu, P. J. Smith, and A. Naguib, "From theory to practice: an overview of MIMO space-time coded wireless systems," IEEE J. Sel. Areas Commun., vol. 21, no. 3, pp. 281-302, 2003.

[4] D. Gesbert, F. Tosato, C. van Rensburg, and F. Kaltenberger, UMTS Long Term Evolution: From Theory to Practice. Wiley and Sons, 2009, ch. Multiple Antenna techniques in LTE. 
[5] J. G. Andrews, A. Ghosh, and R. Muhamed, Fundamentals of WiMAX: Understanding Broadband Wireless Networking. Prentice Hall PTR, Mar. 2007, ch. Multiple-Antenna Techniques.

[6] G. Caire and S. Shamai (Shitz), "On the achievable throughput of a multiantenna Gaussian broadcast channel," IEEE Trans. Inf. Theory, vol. 49, no. 7, pp. 1691-1706, Jul. 2003.

[7] H. Weingarten, Y. Steinberg, and S. Shamai (Shitz), "The capacity region of the Gaussian multiple-input multiple-output broadcast channel,' IEEE Trans. Inf. Theory, vol. 52, no. 9, pp. 3936-3964, Sep. 2006.

[8] C. B. Peel, B. M. Hochwald, and A. L. Swindlehurst, "A vector-perturbation technique for near-capacity multiantenna multiuser communication-part I: channel inversion and regularization," IEEE Trans. Commun., vol. 53, no. 1, pp. 195-202, Jan. 2005.

[9] D. Gesbert, M. Kountouris, R. W. Heath, Jr., C. B. Chae, and T. Sälzer, "From single user to multiuser communications: Shifting the MIMO paradigm," IEEE Signal Process. Mag., vol. 24, no. 5, pp. 36-46, Sep. 2007.

[10] D. Love, R. Heath, V. Lau, D. Gesbert, B. Rao, and M. Andrews, "An overview of limited feedback in wireless communication systems," Selected Areas in Communications, IEEE Journal on, vol. 26, no. 8, pp. $1341-1365$, Oct. 2008.

[11] N. Jindal, "MIMO broadcast channels with finite-rate feedback," IEEE Trans. Inf. Theory, vol. 52, no. 11, pp. 5045-5060, 2006.

[12] N. Jindal and A. Goldsmith, "Dirty-paper coding versus TDMA for MIMO broadcast channels," IEEE Trans. Inf. Theory, vol. 51, no. 5, pp. 1783-1794, May 2005.

[13] T. Al-Naffouri, M. Sharif, and B. Hassibi, "How much does transmit correlation affect the sum-rate scaling of MIMO Gaussian broadcast channels?" IEEE Trans. Commun., vol. 57, no. 2, pp. 562-572, Feb. 2009.

[14] F. Kaltenberger, M. Kountouris, L. S. Cardoso, R. Knopp, and D. Gesbert, "Capacity of linear multi-user MIMO precoding schemes with measured channel data," in Proc. IEEE Intl. Workshop on Signal Processing Advances in Wireless Communications (SPAWC), Recife, Brazil, Jul. 2008.

[15] F. Kaltenberger, L. Bernadó, and T. Zemen, "On the characterization of measured multi-user MIMO channels," in Workshop on Smart Antennas (WSA 2009), Berlin, Germany, Feb. 2009.

[16] P. Viswanath and D. Tse, "Sum capacity of the vector Gaussian broadcast channel and uplink-downlink duality," IEEE Trans. Inf. Theory, vol. 49, no. 8, pp. 1912-1921, Aug. 2003.

[17] K. Huang, R. W. Heath, Jr., and J. G. Andrews, "Limited feedback beamforming over temporally-correlated channels," IEEE Trans. Signal Process., vol. 57, no. 5, pp. 1959-1975, May 2009.

[18] R. de Lacerda, L. S. Cardoso, R. Knopp, M. Debbah, and D. Gesbert, "EMOS platform: real-time capacity estimation of MIMO channels in the UMTS-TDD band," in Proc. International Symposium on Wireless Communication Systems (IWCS), Trondheim, Norway, Oct. 2007.

[19] G. Bauch, J. Anderson, C. Guthy, M. Herdin, J. Nielsen, J. A. Nossek, P. Tejera, and W. Utschick, "Multiuser MIMO channel measurements and performance in a large office environment," in Proc. IEEE Wireless Comm. and Net. Conf., Hong Kong, Mar. 2007, pp. 1900-1905.

[20] G. W. K. Colman and T. J. Willink, "Limited feedback precoding in realistic MIMO channel conditions," in Proc. IEEE Int. Conf. on Comm. (ICC), Glasgow, Scotland, Jun. 2007, pp. 4363-4368.

[21] Q. H. Spencer, J. W. Wallace, C. B. Peel, T. Svantesson, A. L. Swindelhurst, H. Lee, and A. Gumalla, MIMO System Technology for Wireless Communications. CRC Press, Mar. 2006, ch. Performance of Multi-User Spatial Multiplexing with Measured Channel Data.

[22] A. L. Anderson, J. R. Zeidler, and M. A. Jensen, "Stable transmission in the time-varying MIMO broadcast channel," EURASIP Journal on Advances in Signal Processing, 2008.

[23] N. Czink, B. Bandemer, G. V. Vilar, L. Jalloul, and A. Paulraj, "Can multi-user MIMO measurements be done using a single channel sounder?" COST 2100, Lille, France, Tech. Rep. TD(08) 621, Nov. 2008.

[24] J. Koivunen, P. Almers, V.-M. Kolmonen, J. Salmi, A. Richter, F. Tufvesson, P. Suvikunnas, A. F. Molisch, and P. Vainikainen, "Dynamic multilink indoor MIMO measurements at $5.3 \mathrm{GHz}$," in Proc. 2nd European Conference on Antennas and Propagation (EuCAP 2007), Edinburgh, UK, Nov. 2007.

[25] W. Yu and J. M. Cioffi, "Sum capacity of Gaussian vector broadcast channels," IEEE Trans. Inf. Theory, vol. 50, no. 9, pp. 1875-1892, Sep. 2004.

[26] N. Jindal, W. Rhee, S. Vishwanath, S. Jafar, and A. Goldsmith, "Sum power iterative water-filling for multi-antenna Gaussian broadcast channels," IEEE Trans. Inf. Theory, vol. 51, no. 4, pp. 1570-1580, Apr. 2005.
[27] A. Paulraj, D. Gore, and R. Nabar, Introduction to Space-Time Wireless Communications. Cambridge University Press, 2003.

[28] R. Knopp and P. A. Humblet, "Information capacity and power control in single-cell multiuser communications," in Proc. IEEE Int. Conf. on Comm. (ICC), vol. 1, Seattle, CA, Jun. 1995, pp. 331-335.

[29] J. H. Conway, R. H. Hardin, and N. J. A. Sloane, "Packing lines, planes, etc.: Packings in Grassmannian space," Experimental Mathematics, vol. 5, pp. 139-159, 1996.

[30] D. J. Love, R. W. Heath, Jr., and T. Strohmer, "Grassmannian beamforming for multiple-input multiple-output wireless systems," IEEE Trans. Inf. Theory, vol. 49, no. 10, pp. 2735-2747, 2003.

[31] D. J. Love, "Grassmannian subspace packing," Jul. 2004. [Online]. Available: http://cobweb.ecn.purdue.edu/ djlove/grass.html

[32] Philips, "System-level simulation results for channel vector quantisation feedback for MU-MIMO," 3GPP TSG RAN WG1, Tech. Rep. R1063028, Nov. 2006.

[33] F. Kaltenberger, L. Bernadó, and T. Zemen, "Characterization of measured multi-user MIMO channels using the spectral divergence measure," COST 2100, Lille, France, Tech. Rep. TD(08) 640, Nov. 2008.

[34] F. Kaltenberger, M. Kountouris, D. Gesbert, and R. Knopp, "Correlation and capacity of measured multi-user MIMO channels," in Proc. IEEE Intl. Symposium on Personal, Indoor and Mobile Radio Communications (PIMRC), Cannes, France, Sep. 2008.

[35] — , "Performance of multi-user MIMO precoding with limited feedback over measured channels," in Proc. IEEE Global Communications Conference (IEEE GLOBECOM 2008), New Orleans, USA, Nov. 2008.

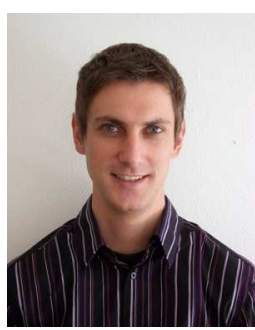

Florian Kaltenberger ( $\left.\mathrm{S}^{\prime} 05-\mathrm{M}^{\prime} 08\right)$ was born in Vienna, Austria, in 1978. He received his Dipl.-Ing. degree and his Ph.D. degree both in technical mathematics from the Vienna University of Technology in 2002 and 2007, respectively.

During the summer of 2001, he held an internship position with British Telecom, BT Exact Technologies in Ipswich, UK, where he was working on mobile video conferencing applications. In 2002, he started as a Research Assistant at the Vienna University of Technology, Institute for Advanced Scientific Computing, working on distributed signal processing algorithms. In 2003, he joined the wireless communications group at the Austrian Research Centers $\mathrm{GmbH}$, where he was working on the development of lowcomplexity smart antenna and MIMO algorithms as well as on the ARC SmartSim real-time hardware channel simulator. Since 2007 he is Research Engineer at Eurecom, Sophia-Antipolis, France where he is working on the Eurecom real-time open-source experimental platform OpenAirInterface.org. His research interests include signal processing for wireless communications, MIMO communication systems, receiver design and implementation, MIMO channel modeling and simulation, and hardware implementation issues.

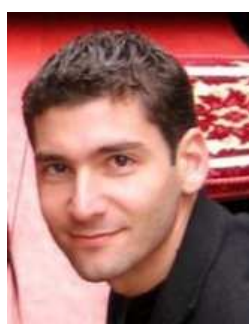

Marios Kountouris (S'04-M'08) received the Dipl.Ing. degree in Electrical and Computer Engineering from the National Technical University of Athens, Greece in 2002 and the M.S. and Ph.D degrees in Electrical Engineering from Ecole Nationale Supérieure des Télécommunications (ENST) Paris, France in 2004 and 2008, respectively.

From February 2008 to May 2009, he has been with the Department of Electrical and Computer Engineering at The University of Texas at Austin as a postdoctoral research associate, working on wireless ad-hoc networks under DARPA's IT-MANET program. Since June 2009 he has been with the Department of Telecommunications at SUPELEC, France where he is currently an Assistant Professor. His research interests include multiuser MIMO communications, wireless ad hoc networks, cross-layer optimization of communication networks, and dynamic resource allocation as well as network information theory. He is a Member of the IEEE and a Professional Engineer of the Technical Chamber of Greece. 
David Gesbert (S'96-M'99-SM'05) is Professeur in the Mobile Communications Dept., Eurecom Institute, France. He obtained the Ph.D degree from Ecole Nationale Supérieure des Télécommunications, France, in 1997. From 1997 to 1999 he has been a research fellow at the Smart Antenna Research Group of the Information Systems Laboratory, Stanford University. In 1999 he was a founding engineer of Iospan Wireless Inc, San Jose, Ca., a startup company pioneering MIMO-OFDM (now Intel).

D. Gesbert has published about 150 papers and several patents all in the area of signal processing, communications, and wireless networks. D. Gesbert is or was a co-editor of several special issues on wireless networks and communications theory, for JSAC (2003, 2007), EURASIP Journal on Applied Signal Processing (2004, 2007), Wireless Communications Magazine (2006). He was an elected member of the IEEE Signal Processing for Communications Technical Committee. He is a member of the French CNRS Expert Committee for "Networks". He's an associate editor for IEEE Transactions on Wireless Communications and the EURASIP Journal on Wireless Communications and Networking. He authored or co-authored papers winning the 2004 IEEE Best Tutorial Paper Award (Communications Society) for a 2003 JSAC paper on MIMO systems, 2005 Best Paper (Young Author) Award for Signal Proc. Society journals, and the Best Paper Award for the 2004 ACM MSWiM workshop.

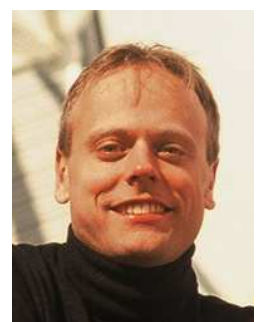

Raymond Knopp was born in Montreal, Canada. He received the B.Eng. (Honours) and the M.Eng. degrees in electrical engineering from McGill University, Montreal, Canada, in 1992 and 1993, respectively. In 1997, received the $\mathrm{PhD}$ degree in communication systems from the Swiss Federal Institute of Technology, Lausanne. During his PhD studies (1993-1997), he was a Research and Teaching Assistant in the Mobile Communications Department of Institut Eurecom, Sophia Antipolis, France. From 1997-2000 he was a research associate in the Mobile Communications Laboratory (LCM-EPFL) of the Communication Systems Department of the Swiss Federal Institute of Technology (EPFL), Lausanne. In 2000 he rejoined the Mobile Communications Department of Institut Eurecom as a Professor.

His current research and teaching interests are in the area of digital communications, software radio architectures, and implementation aspects of signal processing systems. He has published numerous journal and conference articles in these areas. He has participated in collaborative research projects related to wireless communications in the FP5, FP6 and FP7 framework programs as well as French National programs. He is currently Eurecom team leader for the CHORIST FP6 project and workpackage leader in the NEWCOM++ FP7 Network of Excellence. Raymond KNOPP is technical coordinator of the OpenAirInterface.org wireless radio platform initiative. These platforms are used in a variety of collaborative research projects and one-to-one projects with both industrial and academic partners to highlight innovative research ideas in wireless networks through experimentation. 